\title{
Increased intestinal permeability precedes clinical onset of type 1 diabetes
}

\author{
E. Bosi • L. Molteni • M. G. Radaelli • L. Folini • \\ I. Fermo $\cdot$ E. Bazzigaluppi $・$ L. Piemonti • \\ M. R. Pastore • R. Paroni
}

Received: 2 June 2006 / Accepted: 7 August 2006/ Published online: 7 October 2006

(C) Springer-Verlag 2006

\begin{abstract}
Aims/hypothesis Recent observations have shown subclinical intestinal abnormalities in human type 1 diabetes. Whether these are related to the pathogenetic process or secondary to the diabetes remains to be clarified. The aim of this study was to investigate this issue by examining intestinal permeability to sugars in subjects at different stages of type 1 diabetes: preclinical, new-onset and longterm established disease.

Methods Eighty-one subjects with islet autoimmunity (18 preclinical, 28 new-onset and 35 long-term type 1 diabetes) and 40 healthy control subjects were investigated by a lactulose-mannitol test, consisting of oral administration of the two sugars and measurement of their urinary excretion.
\end{abstract}

E. Bosi $(\bowtie) \cdot$ L. Molteni • M. G. Radaelli • L. Folini •

L. Piemonti $\cdot$ M. R. Pastore

General Medicine, Diabetes and Endocrinology Unit,

San Raffaele Scientific Institute

and Vita-Salute San Raffaele University,

Via Olgettina 60,

20132 Milan, Italy

e-mail: bosi.emanuele@hsr.it

I. Fermo

Separative Techniques Unit,

San Raffaele Scientific Institute,

Milan, Italy

E. Bazzigaluppi

Laboraf, Diagnostica e Ricerca San Raffaele,

San Raffaele Scientific Institute

and Vita-Salute San Raffaele University,

Milan, Italy

\section{R. Paroni}

Department of Medicine, Surgery and Dental Science,

San Paolo Hospital, University of Milan,

Milan, Italy
Results All groups of subjects with islet autoimmunity showed an increase in intestinal permeability ( $p \leq 0.009$ vs controls) to the disaccharide lactulose, indicative of a damaged barrier, but a similar permeability to the monosaccharide mannitol (NS vs controls), indicative of an integral surface mucosa; consequently there was an increase in the lactulose:mannitol excretion ratio ( $p \leq 0.025$ vs controls).

Conclusions/interpretation These findings indicate the presence of a subclinical enteropathy associated with type 1 diabetes that is already detectable before clinical onset of the disease, and suggest that the small intestine is an organ participating in the pathogenetic process of type 1 diabetes.

Keywords Type 1 diabetes · Enteropathy · Islet antibodies . Pre-clinical diabetes $\cdot$ Intestinal permeability

$\begin{array}{ll}\text { Abbreviations } \\ \text { BB } & \text { bio-breeding } \\ \text { GADA } & \text { glutamic acid decarboxylase antibody } \\ \text { IA-2A } & \text { protein tyrosine islet antigen-2 antibody } \\ \text { IAA } & \text { insulin autoantibodies } \\ \text { ICA } & \text { islet cell antibody }\end{array}$

\section{Introduction}

A number of recent observations indicate the presence of several intestinal abnormalities in both experimental and human autoimmune diabetes. In the bio-breeding (BB) rat, a model of spontaneous autoimmune diabetes, increased intestinal permeability, mucosal lymphocytic infiltration and morphological changes have been reported [1-3]. More recently, an enteropathy with similar characteristics 
has also been described in the non-obese diabetic mouse [4]. In patients with type 1 diabetes, increased intestinal permeability [5-7], intestinal biopsy-documented signs of enhanced immune activation [8], ultrastructural changes [9] and increased concentrations of circulating zonulin [7] have been shown. In the BB rat, these features, detectable before the onset of clinical diabetes, are thought to be related to the systemic immune disorder; in human type 1 diabetes the natural history and the pathogenetic implications of these alterations remain to be clarified. In order to investigate this issue, we examined the permeability of the intestine to sugars in subjects at the different stages of type 1 diabetes, these being: (1) before clinical onset in subjects with ongoing islet autoimmunity; (2) at the time of clinical diagnosis; and (3) after several years of established disease. The aim was to characterise further the intestinal dysfunction associated with human type 1 diabetes and clarify its natural history in parallel with that of the underlying autoimmune process.

\section{Subjects and methods}

\section{Subjects}

Four groups of individuals were investigated. All patients and controls had no signs or symptoms of enteropathy and all were negative on screening for IgG and IgA transglutaminase antibodies.

Patients with preclinical type 1 diabetes Eighteen firstdegree relatives of patients with type 1 diabetes (nine males, median age 23 years, mean age $20.8 \pm 8.8$ years, range 8-42 years) were recruited through a screening campaign within families with type 1 diabetes, and had two or more autoantibodies among the following: glutamic acid decarboxylase antibody (GADA), protein tyrosine islet antigen-2 antibody (IA-2A) and insulin autoantibodies (IAA), measured by radiobinding assays, and islet cell antibodies (ICA), measured by indirect immunofluorescence. The estimated risk of developing type 1 diabetes in these individuals is $>50 \%$ within 5 years, and therefore they can be considered as representative of subjects with the preclinical phase of the disease.

Patients with new-onset type 1 diabetes These were 28 patients (18 male, median age 28 years, mean age $29.1 \pm$ 8.0 years, range 6-56 years) within 1 year of diagnosis of type 1 diabetes (median duration of diabetes 0.4 months, mean duration $1.5 \pm 2.6$ months, range 0 9 months) based on American Diabetes Association criteria and with either detectable GADA, IA-2A, IAA or ICA.

Patients with long-term type 1 diabetes These were 35 patients (19 male, median age 41 years, mean age $41.0 \pm$ 12.3 years, range 22-66 years) diagnosed as having type 1 diabetes for $\geq 4$ years (median duration of diabetes 20.0 years, mean duration of diabetes $18.7 \pm 7.0$ years, range 4-30 years).

Healthy control subjects These were 40 healthy subjects with no family history of type 1 diabetes and no diabetesrelated autoantibodies (19 male, median age 22 years, mean age $23.7 \pm 9.8$ years, range $9-54$ years).

All subjects participating in the study gave informed consent; the investigations were carried out in accordance with the Declaration of Helsinki as revised in 2000 (http:// www.wma.net/e/policy/b3.htm).

The intestinal permeability test

The permeability of the intestine to sugars was investigated using the lactulose-mannitol test. This consists of the administration of an oral dose of two probes, lactulose and mannitol, and of the measurement of their intestinal uptake by evaluating their urinary excretion in the following $5 \mathrm{~h}$. After an overnight fast and collection of a basal urine sample, subjects drank a 50-ml solution in deionised water of $5 \mathrm{~g}$ lactulose and $2 \mathrm{~g}$ mannitol. Urine was collected during the next $5 \mathrm{~h}$, with the addition of sodium azide as preservative. Two hours after the test was started, subjects were encouraged to drink, and a liberal intake of water was allowed during the next $3 \mathrm{~h}$. The total volume of urine was measured, and a 10-ml sample was stored at $-20^{\circ} \mathrm{C}$ until the assay. Lactulose and mannitol concentrations in urine were measured using a recently developed capillary electrophoresis method [10]. Results are expressed as a percentage of the administered lactulose and mannitol excreted in urine, and as a lactulose:mannitol ratio.

\section{Statistics}

Data are presented as means \pm SD. All statistical and regression analyses were performed using SPSS 10.0 for Windows. Lactulose and mannitol urinary excretion were compared by the non-parametric Kruskal-Wallis test, since normality tests failed. A multiple-comparison model with individual contrast using ANOVA with a post hoc Bonferroni test was applied after log transformation of lactulose and mannitol urinary excretion values. Frequency comparison was performed by the Pearson chi-square test. 

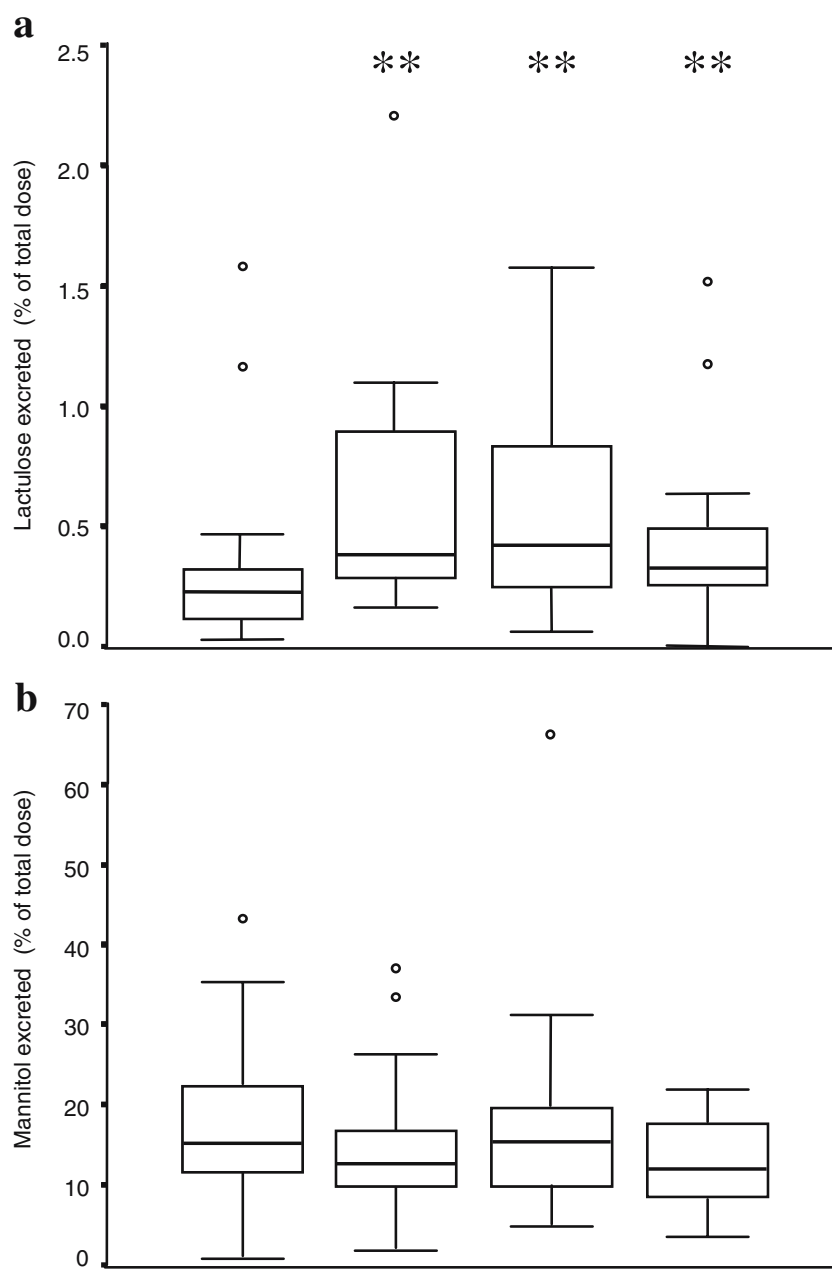

C

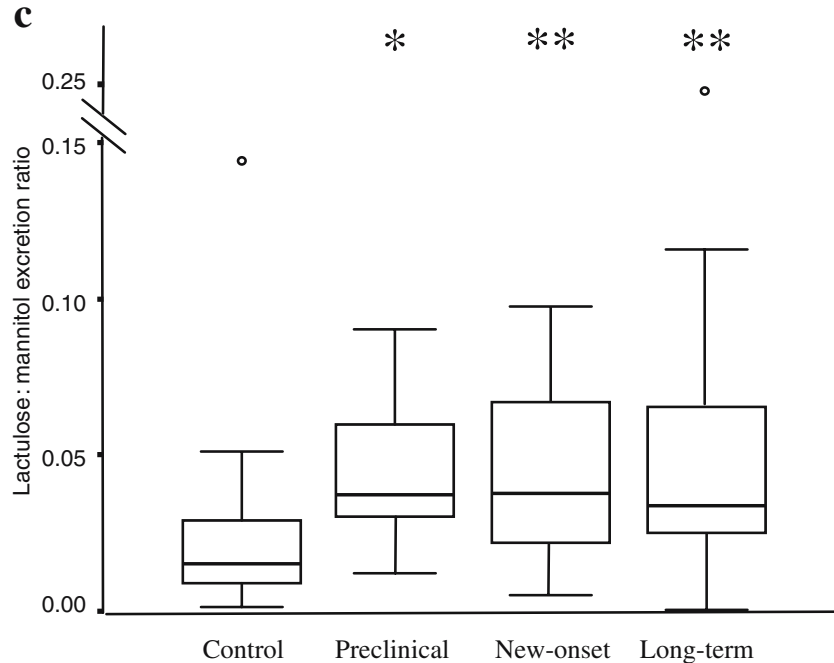

Fig. 1 Percentage of the total administered dose of lactulose (a) and mannitol (b) excreted in the urine and the lactulose:mannitol ratio (c) in healthy controls and subjects with preclinical, new-onset and long-term type 1 diabetes. Data are box and whisker plots with the outliers outside the ranges (o). ${ }^{*} p<0.05$ vs controls; ${ }^{*} p<0.01$ vs controls

\section{Results}

In 12 out of 35 patients with long-term type 1 diabetes and in one with new-onset disease, the presence of glucose in urine interfered with the measurement of lactulose and mannitol and they were excluded from the analysis. No other cases of glycosuria were encountered in the remaining patients. The urinary excretion of lactulose and mannitol and the lactulose:mannitol ratio did not correlate with age or sex, in either patient or control groups.

In comparison with healthy controls, urinary lactulose excretion was significantly increased in all groups of subjects with islet autoimmunity, including preclinical (median 0.350 vs $0.225 \%$ of controls, $p=0.001$ ), new-onset (median $0.498 \%, p=0.002$ vs controls) and long-term type 1 diabetes (median $0.310 \%, p=0.009$ vs controls) (Fig. 1a). Mannitol urinary excretion showed no significant differences between subjects with islet autoimmunity and controls (Fig. 1b). Accordingly, the lactulose:mannitol excretion ratio was significantly increased in all groups of subjects with islet autoimmunity ( $p \leq 0.025$ vs controls) (Fig. 1c). No statistically significant differences in lactulose and mannitol urinary excretion and lactulose:mannitol ratio were observed between the three groups of subjects with the different stages of type 1 diabetes (Fig. 1a-c).

\section{Discussion}

This study demonstrates the presence of a potential, subtle and clinically silent enteropathy associated with type 1 diabetes that is detectable along the whole course of its natural history, independently of the concomitance and duration of glucose abnormalities, suggesting this is a pathogenetic trait of the associated autoimmune process.

The hallmark of this enteropathy is the altered intestinal permeability to sugars, investigated by the lactulosemannitol test. The lactulose-mannitol test is based on the measurement, following their combined oral administration, of the urinary excretion of these two sugars, which are not hydrolysed or actively transported across the intestinal barrier. The sugar alcohol mannitol, which is a small molecule, permeates the intestinal mucosa via a transcellular pathway through the water-filled pores of the cell membrane, whereas the larger disaccharide molecule lactulose uses a paracellular route through the junctional complexes between adjacent enterocytes. An alteration of intestinal anatomical and functional integrity may lead to either a reduced passage of small molecules such as mannitol, as an expression of reduced integrity of the surface mucosa, or an abnormal uptake of large molecules such as lactulose, as an expression of a damaged barrier. 
The most prominent abnormality observed in this study was the increased urinary excretion of lactulose, indicative of a higher intestinal absorption through a paracellular route, and this was uniformly elevated in all stages of the natural history of type 1 diabetes. Conversely, mannitol excretion was similar in subjects with islet autoimmunity and normal controls.

These findings confirm the previous observation of abnormal intestinal permeability in patients with type 1 diabetes $[5,6]$ and indicate the paracellular route as the site of a damaged passive transport across the intestinal barrier. This observation is in accord with a recent report showing in patients with type 1 diabetes and some of their relatives an increased concentration of circulating zonulin, a putative modulator of intestinal permeability, possibly acting on intercellular tight junctions [7]. Our cross-sectional findings indicate that the increased intestinal permeability to lactulose precedes the clinical onset of type 1 diabetes and is detected as uniformly altered during the whole course of the disease, independently of abnormalities secondary to insulin deficiency. Therefore, the evidence for a damaged barrier seems to parallel the autoimmune process rather than the metabolic disorder.

The natural history of intestinal abnormalities associated with human type 1 diabetes looks similar to that observed in diabetes-prone BB rats, in which the increased intestinal permeability precedes hyperglycaemia and lymphocytic insulitis, indicating enteropathy as a very early pathogenetic event [1-3]. In human type 1 diabetes the genesis and significance of the increased intestinal permeability is unknown. Although the increased permeability to lactulose shown here is not direct evidence of an increased permeability to any putative antigenic molecules, which in most cases are larger than this disaccharide, a causative link with autoimmunity can be hypothesised. Thus if the integrity of the intestinal epithelium is primarily compromised by any injury (toxic, infectious, inflammatory, other), an autoimmune reaction could develop as a response to abnormally absorbed macromolecules; conversely, if an immune-mediated process is primarily taking place at the intestinal level regardless of the trigger, mucosal integrity might be compromised as an effect of the associated inflammatory process.

In conclusion, these data provide evidence for the presence of a subclinical enteropathy associated with the autoimmune process underlying type 1 diabetes. These findings indicate that the small intestine may be important in the pathogenesis of type 1 diabetes.

\section{References}

1. Meddings JB, Jarand J, Urbanski SJ, Hardin J, Gall DG (1999) Increased gastrointestinal permeability is an early lesion in the spontaneously diabetic BB rat. Am J Physiol 276:G951-G957

2. Graham S, Courtois P, Malaisse WJ, Rozing J, Scott FW, Mowat AM (2004) Enteropathy precedes type 1 diabetes in the BB rat. Gut 53:1437-1444

3. Neu J, Reverte CM, Mackey AD et al (2005) Changes in intestinal morphology and permeability in the BioBreeding rat before the onset of type 1 diabetes. J Pediatr Gastroenterol Nutr 40:589-595

4. Maurano F, Mazzarella G, Luongo D et al (2005) Small intestinal enteropathy in non-obese diabetic mice fed a diet containing wheat. Diabetologia 48:931-937

5. Mooradian AD, Morley JE, Levine AS, Prigge WF, Gebhard RL (1986) Abnormal intestinal permeability to sugars in diabetes mellitus. Diabetologia 29:221-224

6. Carratu R, Secondulfo M, De Magistris L et al (1999) Altered intestinal permeability to mannitol in diabetes mellitus type I. J Pediatr Gastroenterol Nutr 28:264-269

7. Sapone A, DeMagistris L, Pletzak M et al (2006) Zonulin upregulation is associated with increased gut permeability in subjects with type 1 diabetes and their relatives. Diabetes 55:1443-1449

8. Westerholm-Ormio M, Vaarala O, Pihkala P, Ilonen J, Savilahti E (2003) Immunologic activity in the small intestinal mucosa of pediatric patients with type 1 diabetes. Diabetes 52:2287-2295

9. Secondulfo M, Iafusco D, Carratu R et al (2004) Ultrastructural mucosal alterations and increased intestinal permeability in nonceliac, type I diabetic patients. Dig Liver Dis 36:35-45

10. Paroni R, Fermo I, Molteni L, Folini L, Pastore MR, Bosi E (2006) Lactulose and mannitol intestinal permeability detected by capillary electrophoresis. J Chromatogr B 834:183-187 